\title{
Les neurotransmetteurs de la rétine
}

La détection histochimique des neurotransmetteurs ou de marqueurs de leur métabolisme a permis de préciser les populations cellulaires rétiniennes et leurs connexions. La transmission de l'influx visuel est soumise à une modulation par les cellules amacrines, notamment dopaminergiques, ellesmêmes contrôlées par des neurones GABA-ergiques.

Jeanine Nguyen-Legros Directeur de recherche à l'Inserm $(U-86)$

\section{ADRESSE ET TIRÉS A PART}

J. Nguyen-Legros : laboratoire de neurocytologie oculaire, 15 rue de l'École de Méde- a neurobiologie a bénéficié ces dernières années de l'apport considérable des nouvelles techniques morphofonctionnelles pour l'étude de ces cellules particulières que sont les neurones. Alors que l'histologie du système nerveux reposait depuis plus d'un siècle sur des méthodes figées telles que la coloration de Nissl, ne révélant que la position des corps neuronaux, ou les imprégnations argentiques, révélant de façon aléatoire quelques neurones dans leur totalité, une floraison de méthodes basées sur la physiologie des neurones a vu le jour en l'espace d'une ou deux décennies. Le transport axonal, par exemple, mis en évidence vers les années 60 , a été rapidement utilisé pour le traçage des voies et les études de connexions, en visualisant le déplacement de traceurs dont le choix judicieux peut préférentiellement révéler le sens antéro ou rétrograde.

\section{Révélation histochimique des neurotransmetteurs}

Parmi les techniques d'acquisition récente, la mise en évidence des neurotransmetteurs chimiques utilisés par les neurones pour véhiculer les messages qu'ils échangent entre eux, a été l'une des plus enrichissantes. En effet, la révélation histochimique de la molécule-message utilisée par un neurone sert, d'une part, à le caractériser biochimiquement sur le plan d'une fonction (le transmetteur étant excitateur ou inhibiteur, ou simplement modulateur) et, d'autre part, à établir des analogies, tant morphologiques que fonctionnelles, entre des populations de neurones utilisant la même molécule-message. De plus, la visualisation d'un constituant chimique contenu dans la totalité du neurone qui se trouve ainsi révélé dans son intégralité, avec une définition comparable à celle des imprégnations argentiques mais sans le caractère aléatoire de celles-ci, constitue un merveilleux outil. La forme du neurone avec son arbre dendritique, biochimiquement caractérisé par son neurotransmetteur, donnera des indications sur ses possibilités de connexions locales. Combinée au transport axonal, la mise en évidence du neurotransmetteur montrera les projections spécifiques à distance de ce neurone.

Dans la rétine, comme dans les autres parties du système nerveux central, la localisation des neurotransmetteurs a apporté beaucoup d'informations sur les circuits internes participant à l'élaboration d'un influx visuel dirigé vers les centres.

\section{Structure et circuits rétiniens}

En dépit d'une apparente simplicité, qui a souvent conduit à la proposer comme modèle, la rétine 
est loin d'être parfaitement connue sur le plan de ses circuits internes, qui représentent la somme des influences que peut subir le message visuel entre la phototransduction et l'influx conduit par le nerf optique [1]. Il faut se souvenir, en effet, qu'entre les photorécepteurs rétiniens (cônes et bâtonnets) et les cellu- les ganglionnaires, dont l'ensemble des axones forme le nerf optique, interviennent au moins trois types de neurones, situés dans la couche des grains internes, et dont les propriétés morphologiques et fonctionnelles bien particulières font de la rétine une entité unique au sein du système nerveux central (figure 1).
Au niveau de la conduction centripète de l'influx, un lien direct est établi entre photorécepteurs et cellules ganglionnaires par de petits neurones "bipolaires ", à axone extrêmement court sur lequel on ne peut pas enregistrer de potentiel d'action.

Quant à la modulation de l'influx, deux types de neurones à dispo-

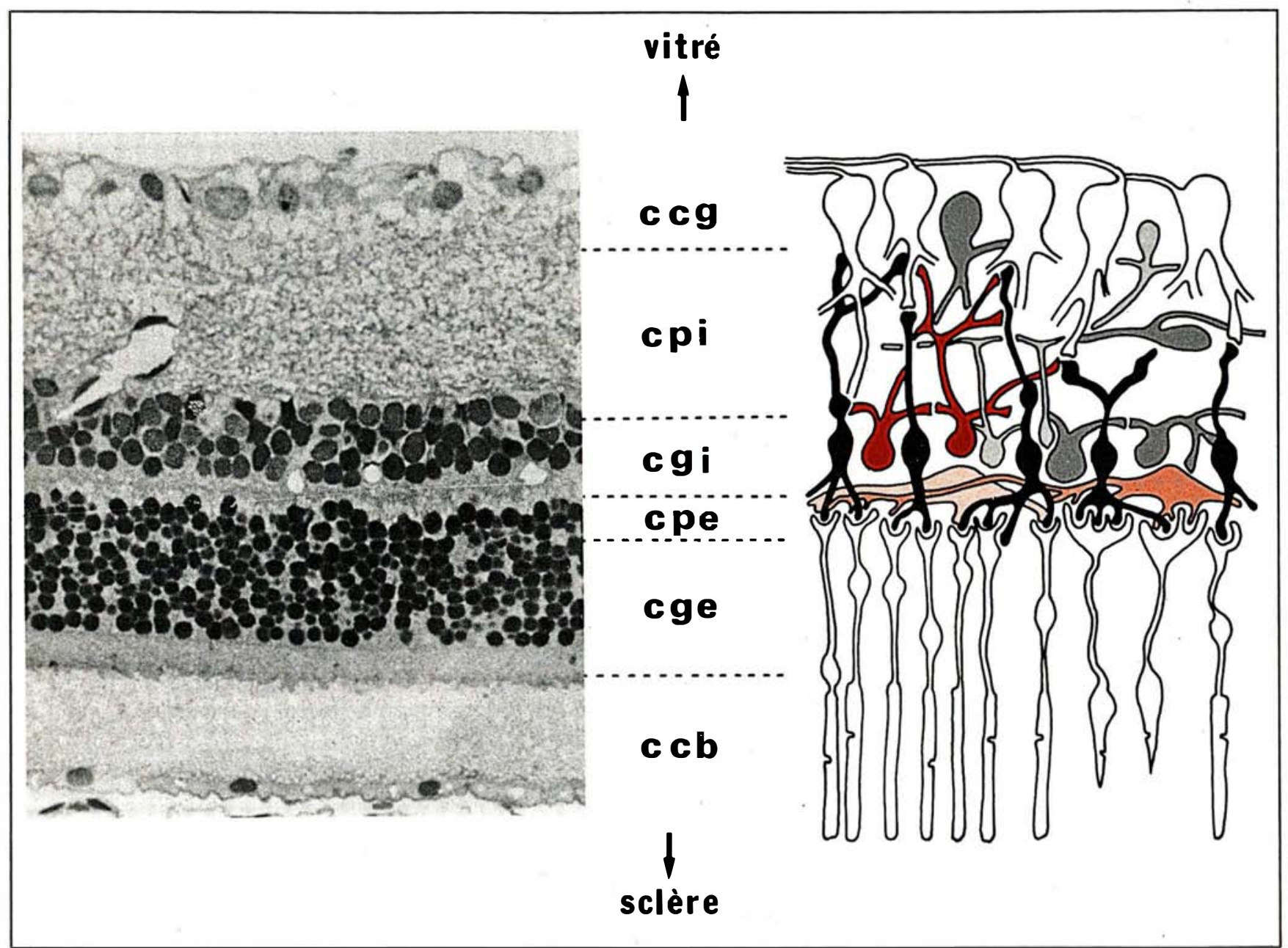

Figure 1. Architecture de la rétine (rat), coupe semi-fine et schéma des connexions. Du póle vitréal au pôle scléral, (1) la couche des cellules ganglionnaires (ccg) contient les cellules ganglionnaires (en blanc), dont les axones forment le nerf optique, et des cellules amacrines déplacées, ici dopaminergiques (en gris foncé) et contenant de la phényléthanolamine-N-méthyltransférase (PNMT, gris clair) ; (2) la couche plexiforme interne (cpi), étage de la deuxième synapse (ganglionnaires-bipolaires) d̀ laquelle participent des cellules amacrines, ici GABAergiques (en rouge), sous forme de dyades, renferme également les connexions des cellules amacrines entre elles, ici dopaminergiques (en gris foncé) et GABAergiques. Cette couche contient également de rares cellules amacrines "interstitielles ", ici dopaminergiques, et reçoit les terminaisons des fibres centrifuges; (3) la couche des grains internes (cgi) contient les corps cellulaires de tous les types d'interneurones rétiniens : cellules bipolaires (en noir), cellules amacrines, ici de trois types, GABAergiques, dopaminergiques et contenant de la PNMT, cellules interplexiformes dopaminergiques (en gris foncé, à droite) contactant les cellules horizontales, à axone (en rose foncé) et sans axone (en rose clair). Les cellules gliales de Müller ne sont pas représentées (noyaux sombres, losangiques sur la photol; (4) la couche plexiforme externe (cpe) est l'étage de la première synapse (photorécepteurs-bipolaires) à laquelle participent les cellules horizontales; (5) la couche des grains externes (cge) renferme les noyaux des photorécepteurs; (6) la couche des cónes et des båtonnets (ccb) est formée des segments internes et externes des photorécepteurs, lieu de la phototransduction. 


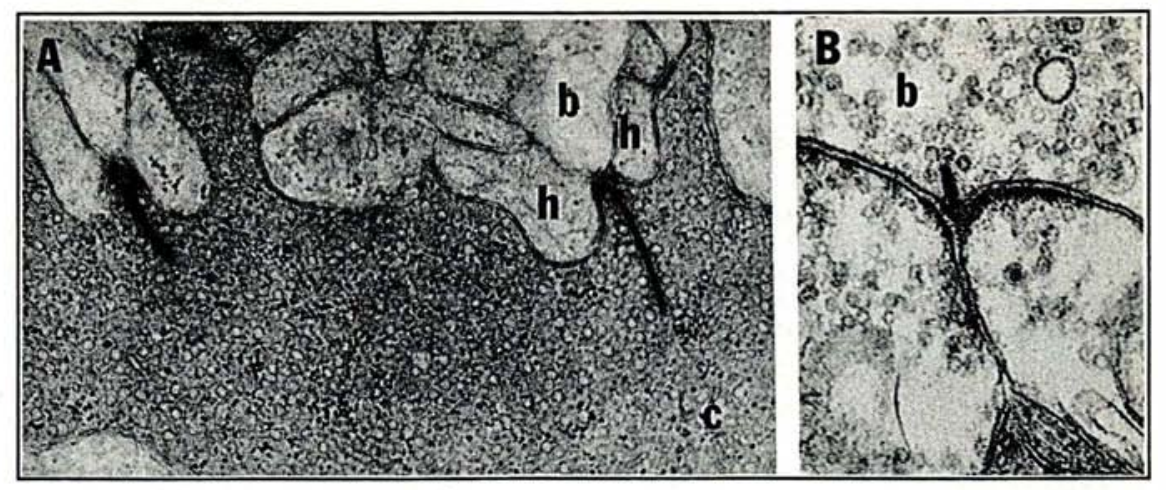

Figure 2. Synapses spécifiques de la chaîno visuello. A. Synapse en triade dans la couche plexiforme externe, ici le pédicule d'un cône (c). En face de chaque ruban synaptique, l'élément central (b) est une dendrite de cellule bipolaire, les deux éléments latéraux (h) sont des prolongements de cellules horizontales. B. Synapse en dyade dans la couche plexiforme interne. La partie pré-synaptique, terminaison axonale d'une cellule bipolaire (b) contient un petit ruban synaptique. Les deux éléments post-synaptiques sont des dendrites de cellules ganglionnaire et amacrine.

sition horizontale interviennent chacun aux deux étages de synapses rétiniennes. Au niveau de la première synapse (photorécepteurs-bipolaires, couche plexiforme externe), les cellules "horizontales " connectent entre eux un certain nombre de photorécepteurs, par des prolongements qui accompagnent les dendrites des cellules bipolaires dans leur invagination $\mathrm{au}$ sein des "pédicules" et "sphérules " synaptiques des photorécepteurs (figure $2 A$ ). Ces cellules sont de deux types, selon qu'elles possèdent ou non un axone, ou plus exactement un télodendron, c'est-à-dire un prolongement donnant naissance à un important bouquet terminal mais

Les jonctions électrotoniques ou synapses électriques sont des points de contact entre deux neurones, où les membranes cellulaires apposées comportent des canaux ioniques $\left(\mathrm{Na}^{+}\right.$, $\mathrm{K}^{+}$) établissant un pontage entre les deux cellules. La perméabilité de ces canaux assure le passage des ions (et petites molécules) d'une cellule à l'autre, et donc une propagation rapide de l'influx, qui peut être bidirectionnelle.

Les synapses chimiques sont des points de contact entre ne générant pas de potentiel d'action et semblant fonctionner pour son propre compte, indépendamment du corps cellulaire. Les cellules horizontales sont couplées entre elles par des jonctions électrotoniques* qui, selon leur état de perméabilité ionique, vont réguler la taille des champs récepteurs des cellules bipolaires.

Au niveau de la seconde synapse (bipolaires-ganglionnaires, couche plexiforme interne), les cellules " amacrines " (étymologiquement : sans axone) prennent part aux synapses bipolaires-ganglionnaires sous forme de dyades (figure $2 B$ ) et sont connectées entre elles par des synapses conventionnelles. Des sous-groupes morphofonctionnels

deux neurones, où une molécule, le neurotransmetteur, libérée dans la fente synaptique par exocytose de vésicules à la face membranaire du neurone présynaptique, va agir sur des récepteurs de la face membranaire du neurone post-synaptique. L'interaction du transmetteur avec le récepteur va, par une cascade de réactions chimiques aboutissant à l'ouverture des canaux ioniques, transmettre, avec une certaine latence, un influx nécessairement unidirectionnel.
4. Brecha $N$, Johnson D, Lieberman AR, navelas J. Substance P-containing retinal glion cells and their central projections in the rabbit. Colloque Inserm : Structure et fonction dans le systeme visuel. Lyon 10-12 sept 1986. 
de cellules amacrines peuvent être distingués par le niveau de la couche plexiforme interne qu'atteignent leurs dendrites et donc leurs connexions. Celles dont les connexions se font dans la souscouche " a " (moitié externe ou sclérale de la plexiforme interne) interviennent au niveau des circuits "OFF "** de la conduction visuelle, c'est-à-dire des cellules bipolaires et ganglionnaires qui s'hyperpolarisent sous l'effet de la lumière. Celles qui atteignent la sous-couche " b " (moitié interne ou vitréale de la plexiforme interne) prennent part aux circuits "ON "** c'est-à-dire ceux des cellules bipolaires et ganglionnaires qui se dépolarisent sous l'effet de la lumière. Des cellules amacrines dites "déplacées " dans la couche des ganglionnaires, participent plus fréquemment aux synapses de la sous-couche $b$ [2]. Ce schéma, simplifié pour les besoins de l'exposé, ne traduit que très imparfaitement la complexité du fonctionnement interne de la rétine, dont les circuits $\mathrm{ON}$ et $\mathrm{OFF}$ ne sont en réalité pas aussi clairement séparés et sont capables d'interactions mutuelles. Des neurones plus récemment découverts, les cellules "interplexiformes ", ont un rôle encore mal compris. Situées parmi les grains internes, au niveau des cellules amacrines, elles ont des prolongements qui atteignent également les deux couches plexiformes et semblent conduire un influx centrifuge (en sens inverse de l'influx visuel) provenant soit d'un mécanisme de feedback interne à la rétine, soit de "fibres centrifuges " issues de noyaux encéphaliques encore mal identifiés chez les mammiferes [3].

\section{L'électrorétinogramme}

La rétine constitue donc un ensemble neuronal bien spécifique, ayant une activité propre, qui peut être enregistrée globalement sous la forme d'un électrorétinogramme (ERG) (figure 3).

\footnotetext{
- Voir encadré p. 200.

- Voir encadré p. 202.

$\mathrm{m} / \mathrm{s} n^{\circ} 4$ vol. 3, avril 87
}

Dans ce tracé, l'onde négative a représente l'activité des photorécepteurs, l'onde positive b, attribuée successivement à plusieurs types cellulaires, représente plus probablement une somme d'activités dues aux circuits internes de la rétine. Les potentiels oscillatoires (PO) sont plus spécifiquement en rapport avec l'activité des cellules amacrines. Ce sont l'onde b et les PO qui vont être modifiés le plus souvent dans les cas de déficiences pathologiques ou d'interventions pharmacologiques sur la conduction et la modulation internes de l'influx visuel. En effet, les circuits internes de la rétine fonctionnent essentiellement grâce à des synapses de type chimique*.

\section{Neurotransmetteurs}

Curieusement on ne sait que peu de choses concernant l'identité des transmetteurs impliqués dans la conduction centripète de l'influx. $\mathrm{Au}$ niveau des photorécepteurs et des cellules bipolaires hyperpolarisantes, il s'agirait d'acides aminés excitateurs du type glutamate/aspartate dont l'efflux continuel, du fait de la dépolarisation permanente de ces cellules, ne facilite certes pas la localisation. $\mathrm{Au}$ niveau des cellules ganglionnaires, seule une petite partie de la population, dont on peut visualiser les projections centrales, semble contenir de la substance $P$, chez le lapin [4]. C'est au niveau des neurones à connexions transversales, et principalement au niveau des amacrines, que l'on a pu localiser avec précision un certain nombre de neurotransmetteurs/modulateurs.

Outre la cohorte des peptides neuroactifs (enképhalines, substance $P$, somatostatine, VIP, cholécystokinine, neurotensine, histamine, neuropeptide $Y$, etc.) qui sont présents et parfois co-localisés

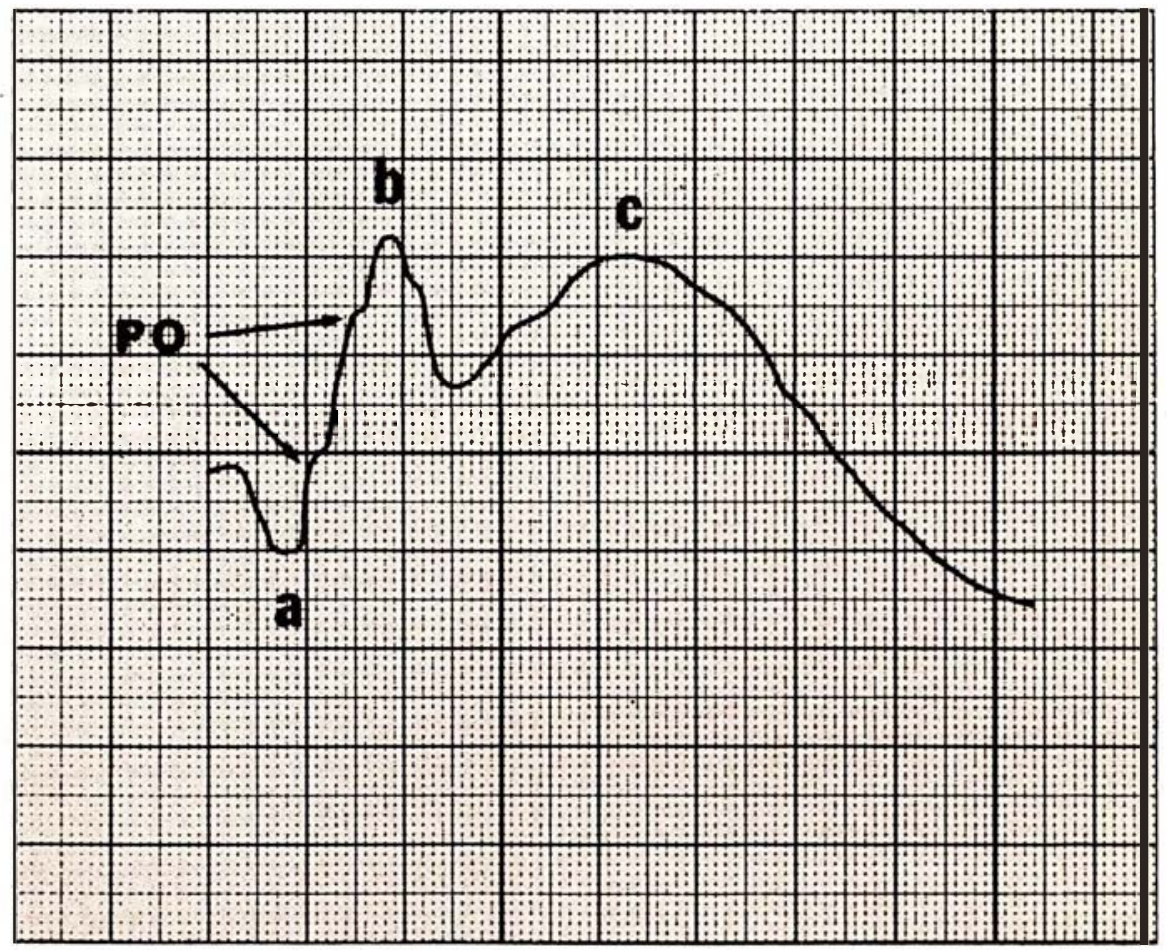

Figure 3. Electrorétinogramme d'un sujot normal. L'onde négative (a) représente l'activité des photorécepteurs, l'onde positive (b) représente une somme d'activités des cellules établissant les circuits internes de la rétine. Les potentiels oscillatoires (PO) sont plus spécialement dus aux cellules amacrines. L'onde (c) serait due à l'épithélium pigmentaire. 


\section{RÉFÉRENCES}

5. Ehinger B. Functional role of dopamine in the retina. In : Osborne $\mathrm{N}$, Chader $\mathrm{G}$, eds. Progress in retinal research. Oxford : Pergamon Press, 1983 ; vol. II : 213-32.

6. Dowling JE. Dopamine : a retinal neuromodulator. Trends in Neuroscience 1986 ; 9 : 236-40.

7. Morgan WW. GABA : a potential neurotransmitter in retina. In : Morgan WW, ed. Retinal transmitters and modulators : a model for the brain. Boca Raton : CRC Press, 1985 ; vol II : 63-96.

8. Nguyen-Legros J, Martin-Martinelli E, Simon A. The significance of PNMT. immunoreactivity in some cells of the rat retina. In : Ehinger B, Agardh E, eds. Retinal signal systems, degenerations and transplants. Amsterdam : Elsevier, 1986 : 173-6.

9. Descarries L, Beaudet A. The use of radioautography for investigating transmitterspecific neurons. In : Björklund $\mathrm{A}$, Hökfelt $\mathrm{T}$, eds. Methods in chemical neuroanatomy. Amsterdam : Elsevier, 1983 ; vol I : 286-364.

10. Saez JC, Spray DC, Nairn AC, Hertzberg E, Greengard P, Bennet MVL. cAMP increases junctional conductance and stimulates phosphorylation of the $27 \mathrm{kDa}$ principal gap junction polypeptide. Proc Natl Acad Sci USA 1986 ; 83 : 2473-7.

11. Jensen RJ, Daw NW. Effects of dopamine and its agonists and antagonists on the receptive field properties of ganglion cells in the rab-
Les photorécepteurs de la rétine sont dépolarisés, c'est-àdire excités, à l'obscurité (courant positif d'obscurité). Ils libèrent continuellement des molécules de neurotransmetteur vers les neurones de second ordre (bipolaires et horizontales) avec lesquels ils sont connectés. L'effet de la lumière est une hyperpolarisation (courant négatif) qui module la libération du transmetteur.

Les cellules bipolaires ont deux types de réponse à la lumière : les unes répondent par une dépolarisation, ce sont les bipolaires dépolarisantes ou cellules $\mathrm{ON}$; les autres répondent par une hyperpolarisation, ce sont les bipolaires hyperpolarisantes ou cellules OFF.

Il existe en réalité un antagonisme entre les réponses du centre et de la périphérie du champ récepteur, celui-ci étant défini comme l'aire rétinienne dans laquelle le stimulus lumineux produit un changement de potentiel de membrane d'une cellule bipolaire.

Le centre du champ récepteur, qui répond par une dépolarisation pour les cellules ON (ou centre-ON) et par une hyperpolarisation pour les cellules OFF (ou centre-OFF), correspond aux connexions directes entre photorécepteurs et bipolaires. La périphérie du champ récepteur qui, inversement, répond par une hyperpolarisation pour les cellules ON et par une dépolarisation pour les cellules OFF, correspond aux connexions établies par l'intermédiaire des cellules horizontales. Un mécanisme sensiblement analogue se reproduit au niveau de la seconde synapse bipolaire-ganglionnaire, avec l'intermédiaire des cellules amacrines. Les étages de la plexiforme interne où se font les connexions entre cellules ON (sous-couche $b$, interne) et cellules OFF (sous-couche a, externe) correspondent aux circuits ON et OFF. entre eux ou avec d'autres médiateurs dans les cellules amacrines, ces cellules comprennent une grande variété de sous-groupes qui peuvent contenir de l'acétylcholine, des monoamines, des acides aminés et de nombreuses hormones. Parmi ces substances, les plus anciennement et les mieux connues sont la dopamine $[5,6]$ et l'acide gamma-amino-butyrique ou GABA. C'est de ces deux types de neurones que nous allons parler plus précisément.

\section{La dopamine}

La dopamine qui a pu être localisée dans les cellules amacrines grâce à la technique d'histochimie de fluorescence de Falck-Hillarp, représente dans la rétine la seule catécholamine dont le rôle neurotransmetteur/modulateur soit établi. En effet, la noradrénaline et l'adrénaline n'existent qu'en très faible quantité et n'ont pu être localisées dans la rétine, bien qu'il existe une population de cellules amacrines immuno-réactives à la phénylétha nolamine- $\mathrm{N}$-méthyltransférase, enzyme de synthèse de l'adrénaline, dont la signification reste obscure.

La dopamine se trouve localisée dans deux types d'interneurones rétiniens : des cellules amacrines dites " interamacrines ", car elles ne font synapse qu'avec d'autres cellules amacrines dans la couche plexiforme interne, et des cellules interplexiformes, dont nous avons vu qu'elles établissaient un circuit récurrent de la plexiforme interne à la plexiforme externe. Ces deux types de cellules présentent, à un prolongement à orientation sclérale près, les mêmes caractères morphologiques qui permettent de les distinguer des autres cellules amacrines : grande taille du périkaryon et arbre dendritique étoilé 
et plat, localisé dans la partie la plus externe de la couche plexiforme interne (figure 4A). Les dendrites sont spécialement longues et déterminent des champs dendritiques très vastes (plus d'un millimètre de diamètre). La densité des cellules dopaminergiques (environ $20 / \mathrm{mm}^{2}$ ) fait que ces champs dendritiques se chevauchent largement et que la rétine entière est couverte par un plexus de prolongements dopaminergiques, au niveau des circuits OFF de la plexiforme interne. Les prolongements externes des cellules interplexiformes, en revanche, ne recouvrent pas la totalité de la rétine, du moins chez les mammifères (ils sont plus nombreux chez les poissons, où toutes les cellules dopaminergiques sont considérées comme interplexiformes). Ils établissent dans cette couche des relations synaptiques avec les cellules horizontales, qui portent des récepteurs dopaminergiques, et des relations de proximité avec les parois vasculaires. Il est à remarquer qu'à aucun des deux étages synaptiques de la rétine les cellules dopaminergiques n'entrent en contact avec les neurones assurant la conduction centripète de l'influx

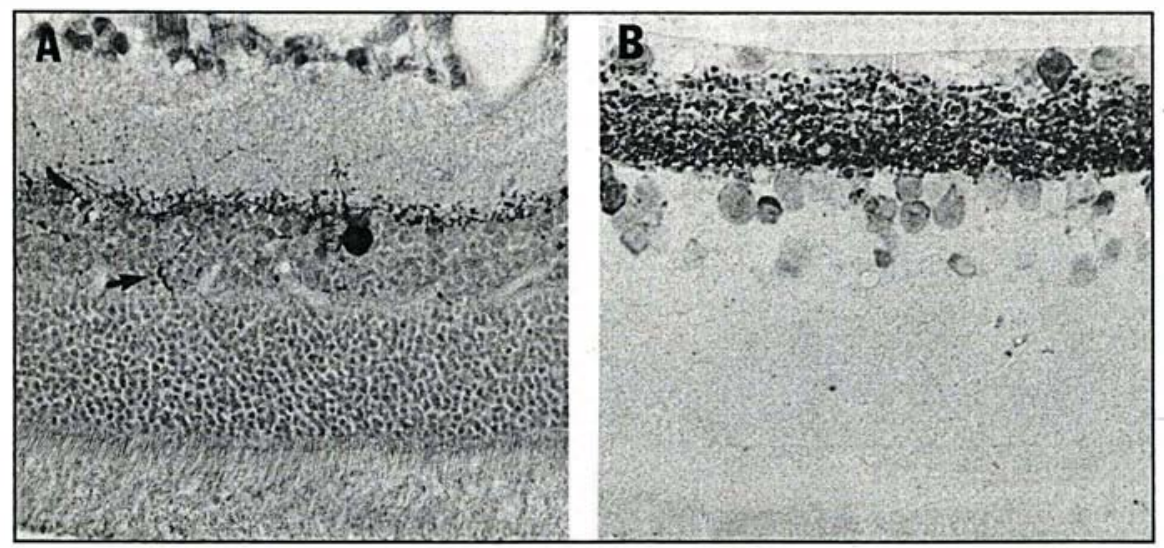

Figure 4. Mise on évidence immunohistochimique des cellules amacrines. A. Dopaminergiques (par la tyrosine-hydroxylase). B. GABAergiques.

Les cellules dopaminergiques sont grosses et peu nombreuses lenviron $5 \%$ des amacrines). Elles épanouissent leurs prolongements au niveau le plus externe de la couche plexiforme interne (sous-couche b). On peut observer quelques prolongements à direction sclérale, provenant de cellules interplexiformes (flèche). Les cellules GABAergiques, de taille variable, sont très nombreuses et se ramifient à tous les niveaux de la couche plexiforme interne. Les cellules immunoréactives de la couche des ganglionnaires sont des amacrines déplacées. (Seule la coupe traitée par l'anti-TH a été contre-colorée pour montrer les couches de la rétine).

$m / s n^{\circ} 4$ ool. 3, auril 87 récepteurs dopaminergiques. Le support morphologique de cette action serait logiquement constitué par les cellules interplexiformes dopaminergiques. C'est en effet ce qui se passe chez les poissons, mais chez la tortue, où l'effet physiologique de la dopamine et la présence de récepteurs sur les cellules horizontales a été démontrée, l'existence de cellules interplexiformes dopaminergiques n'a pas pu être observée jusqu'à présent. Faut-il voir dans cette constatation une carence des méthodes de démonstration ou une preuve de l'action à distance de la dopamine ? La question reste ouverte, bien que l'action de la dopamine en dehors de sites synaptiques semble admise au niveau de l'encéphale [9].

L'action de la dopamine sur ses récepteurs post-synaptiques (couplés à l'adénylate-cyclase) est relayée par un second messager, l'AMP-cyclique (AMPc) qui conduit à la phosphorylation d'une protéine. La protéine $27 \mathrm{kD}$, principal constituant des jonctions électrotoniques, est précisément susceptible d'être phosphorylée par une protéine kinase AMPcdépendante, cette phosphorylation modifiant la perméabilité des canaux ioniques de la jonction [10]. Ce mécanisme serait celui qui agit au niveau des cellules horizontales.

La dopamine exerce également une action inhibitrice sur les cellules ganglionnaires. Or, il n'y a pas non plus de contact direct entre les amacrines dopaminergiques et les cellules ganglionnaires, et des récepteurs à la dopamine n'ont pas été localisés jusqu'à présent sur ces cellules. Il faut donc, dans ce cas, accepter que la dopamine agisse par l'intermédiaire d'un autre interneurone qui contacte la chaîne visuelle. Ce neurone, une cellule amacrine, pourrait être GABAergique ou glycinergique [11].

\section{Le GABA}

Le GABA est très abondant dans la rétine. Il est localisé dans un grand nombre de cellules amacri- 
nes et d'amacrines déplacées qui se ramifient à tous les niveaux de la plexiforme interne (figure $4 B$ ). Les prolongements des cellules GABAergiques participent effectivement aux dyades de la plexiforme interne et reçoivent de ce fait un message de la chaîne visuelle, alors qu'elles passent leur propre message à d'autres cellules amacrines (parmi lesquelles très vraisemblablement les amacrines dopaminergiques) et aux cellules ganglionnaires.

L'activation-même du métabolisme de la dopamine par la lumière, c'est-à-dire un mécanisme dépendant de la photoréception (qui est effectivement perturbé chez les rats RCS ayant une dégénérescence génétique des bâtonnets), alors qu'aucune connexion n'existe entre la chaîne visuelle et les neurones dopaminergiques, montre qu'un neurone intermédiaire intervient à ce niveau. Cet interneurone est très vraisemblablement GABAergique. En effet, la synthèse et la libération de dopamine sont ralenties par le muscimol (agoniste du GABA). Cette action est réversée par la picrotoxine et la bicuculline (bloquant des récepteurs au GABA), ce qui suggère la présence de récepteurs GABAergiques sur les neurones dopaminergiques.

L'interposition d'un neurone GABAergique entre la chaîne visuelle et les cellules interplexiformes dopaminergiques se retrouve également au niveau de l'action de la dopamine sur les cellules horizontales. En effet, la bicuculline, comme la dopamine, limite

\section{RÉFÉRENCES}

12. Bodis-Wollner I, Mitra S, Bobak P, Guillery S, Mylin L. Low frequency distorsion in spatio-temporal threshold surface in Parkinson's disease. Invest Ophthalmol Vis Sci 1984 ; suppl $25: 31$.

13. Domenici L, Trimarchi C, Piccolino $M$, Fiorentini A, Maffei L. Dopaminergic drugs improve human visual contrast sensitivity. Hum Neurobiol 1985 ; 4 : 195-7.

14. Wong C, Ishibashi T, Tucker G, Hamasaki $D$. Response of the pigmented rabbit retina to NMPTP, a chemical inducer of Parkinsonism. Exp Eye Res 1986 ; 40 : 509-19.

15. Dearry A, Burnside B. Dopaminergic regulation of cone retino-motor movement is isolated teleost retina. I. Induction of cone contraction is mediated by D2 receptors. J Neurochem $1986 ; 46$ : 1006-21.

16. Mariani AP, Neff NH, Hadjiconstantinou M. 1-methyl-4-phenyl-1,2,3,6-tetrahydropyridine (MPTP) treatment decreases dopamine and increase lipofuscin in mouse retina. Neurosci Lett 1986 ; 72 : 221-6.

17. Northington FK, Hamill RW, Banerjee SP. Dopamine-stimulated adenylate cyclase and tyrosine hydroxylase in diabetic rat retina. Brain Res 1985 ; 337 : 151-4.

18. Kato S, Sugawara K, Negishi K. The dopaminergic cells in the dystrophic $(\mathrm{C} 3 \mathrm{H})$ mouse retina : a histofluorescence study. Exp Eye Res 1981 ; 33 : 577-9.

19. Osborne NN. Tyrosine-hydroxylase immunoreactivity in retinas from a canine model for Batten-Stengel disease. Naturwissenschaften 1985 ;

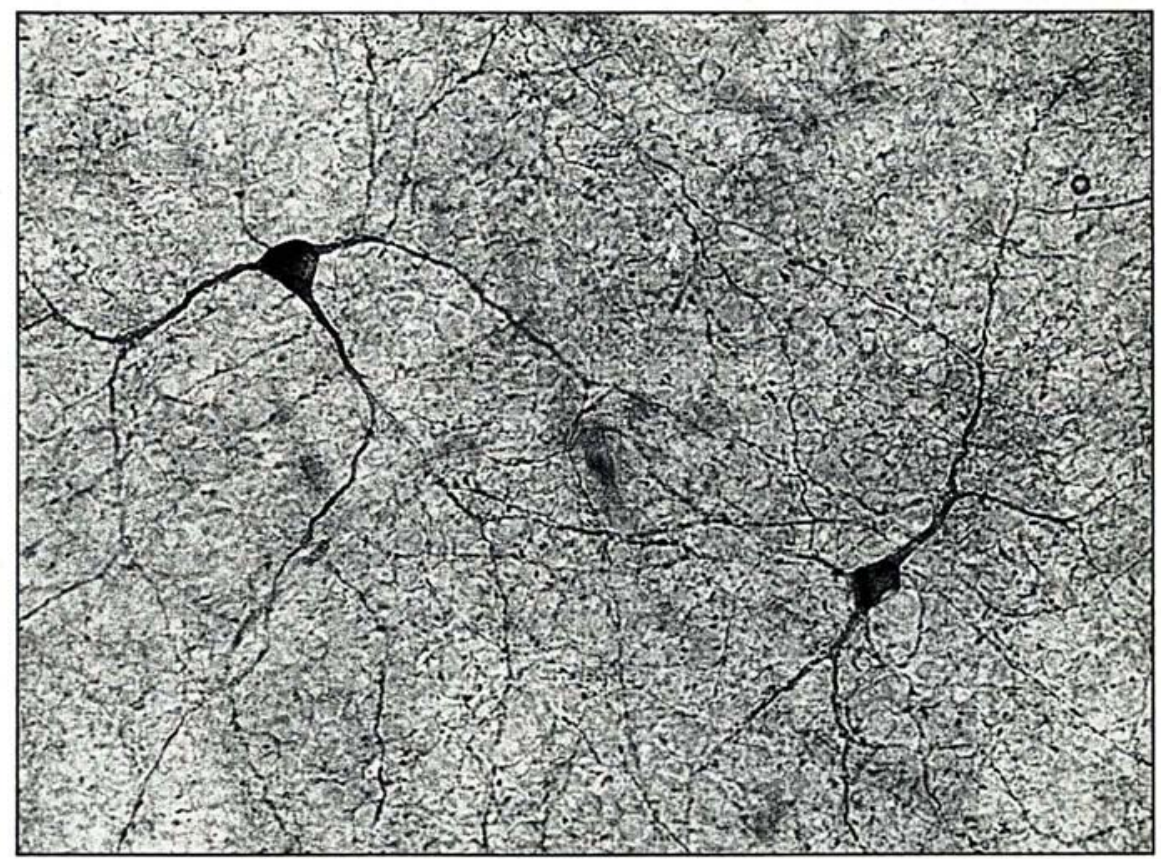

Figure 5. Neurones dopaminergiques de la rétine humaine mis en évidence par un anticorps anti-tyrosine-hydroxylase sur rétine à plat. Pour la morphologie, la rétine présente l'avantage de pouvoir être traitée entière, comme une coupe épaisse (200 micromètres). Elle reste ainsi intacte, avec ses trois dimensions observables sur une seule lame et fournit une préparation fidèle et très utile pour l'étude de sa topographie, surtout en ce qui concerne les circuits internes dont les neurones sont entièrement intra-rétiniens. Ici, une rétine humaine a été traitée dans sa totalité par un anticorps anti-tyrosinehydroxylase pour montrer les neurones dopaminergiques. On peut ainsi apprécier la morphologie, les dimensions et le degré de recouvrement des champs dendritiques. Des cartographies peuvent être réalisées sur ce type de préparation, indiquant la distribution et la densité précises des neurones chimiquement caractérisés par leur neurotransmetteur (deux neurones dopaminergiques $\times$ 4001. 
le couplage des cellules horizontales, ce qui signifie que le blocage des récepteurs au GABA (présumés sur les interplexiformes dopaminergiques) lève le contrôle inhibiteur exercé par le GABA sur les interplexiformes. Il y a alors libération de dopamine, provoquant la fermeture des canaux ioniques des jonctions selon le mécanisme précédemment décrit. Là encore, l'activation du neurone dopaminergique apparaît liée à un phénomène de désinhibition par inactivation du GABA qui le contrôle.

\section{Dopamine et}

pathologie rétinienne

La pathologie de la neurotransmission rétinienne est encore mal connue, et ce pour plusieurs raisons : la neurobiologie des transmetteurs est relativement récente, au niveau rétinien, et l'élément de diagnostic indiquant un dysfonctionnement des circuits rétiniens, l'électrorétinogramme, ne donne qu'une information globale sur l'activité de ces circuits.

On constate cependant, chez les Parkinsoniens par exemple, un retard des potentiels évoqués visuels qui serait dû à une déficience des circuits internes de la rétine, puisqu'il est accompagné d'une décroissance de l'amplitude de l'onde b de l'électrorétinogramme [12]. On retrouve ces deux mêmes anomalies chez les animaux modèles (rat, lapin) traités par l'alpha-méthyl-paratyrosine (inhibiteur compétitif de la synthèse de dopamine), la 6-hydroxydopamine (neurotoxique des cellules dopaminergiques) ou la 1-méthyl-4-phényl-1,2,3,6tétrahydropyridine (MPTP, inducteur de la maladie de Parkinson). Il n'y a pas, chez ces animaux, de retard des potentiels évoqués lorsqu'ils sont obtenus à partir de la stimulation directe du nerf optique, ce qui confirme une atteinte spécifiquement rétinienne. Inversement, on a pu montrer récemment que, d'une part, la prise de L-DOPA corrigeait ces troubles chez les Parkinsoniens et que, d'autre part, la prise de médicaments anti-parkinsoniens par des $m / s n^{\circ} 4$ ool. 3, aoril 87 sujets sains améliorait leur sensibilité aux contrastes, sensibilité qui est diminuée chez les malades, dans les mêmes gammes de fréquence [13]. Toutes ces observations évoquent une pathologie des neurones dopaminergiques de la rétine.

Des études sont en cours, au niveau biochimique* (dosage de la dopamine post-mortem dans les rétines de malades) et morphologique** (structure, densité et distribution des neurones dopaminergiques marqués par la tyrosine hydroxylase) (figure 5). Des conclusions ne peuvent encore être dégagées. Il semble, cependant, que les neurones dopaminergiques de la rétine ne subissent pas une dégénérescence comparable à celle du Locus niger. Une étude récente en microscopie électronique, menée chez le lapin traité par le MPTP, montre qu'un certain nombre de neurones rétiniens contiennent des inclusions intranucléaires identiques à celles observées dans l'encéphale et retenues comme indicateur d'un vieillissement cellulaire. Parmi les neurones contenant ces inclusions, se trouve un nombre important de cellules amacrines mais, curieusement, pas celles que l'on pourrait identifier comme dopaminergiques par leur grande taille. L'hypothèse faite est que, du fait du ralentissement de son métabolisme, la dopamine cesserait d'exercer son inhibition sur les neurones post-synaptiques. Ces neurones deviendraient donc hyperactifs, ce qui entraînerait leur vieillissement prématuré et le développement des inclusions intra-nucléaires [14].

Un autre aspect de l'action de la dopamine dans la rétine est le rôle qu'elle joue dans la régulation des réactions rétinomotrices (changement de dimension des segments internes des photorécepteurs selon les conditions d'éclairement) chez les vertébrés inférieurs [15]. Chez les mammiferes, où ces réactions n'ont pas lieu, il semble que la

\footnotetext{
- Unité 289 de l'Inserm.

- Dans notre laboratoire.
}

dopamine régule le renouvellement des disques des segments externes des photorécepteurs. Les souris traitées par le MPTP accumulent en effet rapidement de la lipofuscine. Cette lipofuscine, qui se trouve dans l'épithélium pigmentaire de la rétine, est le résultat de l'activité de phagocytose par cet épithélium des segments externes desquamés par les photorécepteurs. Elle s'accumule normalement au cours du vieillissement, avec un rythme qui serait contrôlé par la dopamine. Le résultat du traitement par le MPTP est, là encore, un phénomène de vieillissement accéléré, dû à une hyperactivité résultant de la levée du contrôle inhibiteur exercé par la dopamine [16]. Ce phénomène pourrait être une composante des troubles visuels observés chez les Parkinsoniens. Les diabétiques montrent de même, à un stade précoce du développement de la rétinopathie, des modifications de l'électrorétinogramme au niveau des PO, pouvant être imputées à un dysfonctionnement des cellules amacrines. Le modèle animal du rat rendu diabétique par la streptozotocine présente les mêmes troubles. Le métabolisme de la dopamine, étudié chez ces rats, se révèle très perturbé. L'activité de la tyrosine hydroxylase et, par conséquent, la synthèse de dopamine, sont diminuées, en même temps qu'est augmentée la libération de dopamine. Ceci entraîne une hypersensibilité des récepteurs dopaminergiques post-synaptiques, mesurée par une augmentation de l'activité adénylate-cyclasique dopamino-sensible [17]. Aucune étude n'a encore été faite sur la rétine des malades.

En définitive, à travers ses différents modes d'action au niveau des cellules horizontales, ganglionnaires et photoréceptrices, la dopamine semble jouer un rôle dans le phénomène d'adaptation de l'obscurité à la lumière. Le métabolisme entier de la dopamine, synthèse, libération, recapture, est activé par la lumière alors que l'obscurité favorise son stockage. La libération de dopa- 
mine induite par la lumière ne déclenche que des réactions inhibitrices : réduction du champ récepteur au niveau des cellules horizontales, diminution de l'activité électrique au niveau des cellules ganglionnaires, ralentissement de la synthèse protéique au niveau des photorécepteurs.

Il n'en reste pas moins étonnant que, s'il est indiscutable que le métabolisme de la dopamine est hautement dépendant des conditions d'éclairement, le développement, la morphologie et la densité des cellules dopaminergiques, sont très peu affectés par ces conditions. C'est ainsi que le système dopaminergique rétinien se développe normalement chez les rongeurs mutants sans bâtonnets, rats RCS (Royal College of Surgeons) et souris portant la mutation rd (retinal degeneration) [18], et que ce système semble normal chez des chiens atteints de céroïde-lipofuscinose, apparemment aveugles [19]. Bien plus, il persiste, avec un développement qui paraît normal, chez le Protée, batracien cavernicole génétiquement aveugle depuis plusieurs millions d'années dont I'œil enkysté, dépourvu de milieux transparents, ne comporte qu'une rétine résiduelle. Il est vrai que ces animaux ont gardé une certaine photosensibilité se manifestant par un comportement de fuite devant l'éclairement. On peut supposer qu'une libération de dopamine est déclenchée par cet éclairement et contribue à la mise en jeu du comportement de fuite. On peut se demander, cependant, quelle est la signification de la conservation d'un tel mécanisme chez un animal adapté depuis si longtemps à la vie souterraine. On serait tenté de penser, là encore, qu'il y a une discordance entre l'état des neurones et le métabolisme de leur transmetteur. Malheureusement, le petit œil de 300 micromètres de diamètre du Protée ne se prête guère aux travaux biochimiques. Cette dernière observation n'est pas sans jeter un certain trouble sur l'importance admise du rôle de la dopamine dans la conduction de l'influx visuel. Il semble bien, comme le fait remarquer Dowling [6], que la dopamine doive être considérée comme neuro-modulateur plutôt que transmetteur et que son rôle le plus probable soit lié à la régulation de certains rythmes d'activité rétinienne en rapport avec l'éclairement

\section{Summary}

The retina, like the other parts of the central nervous system, has taken a large benefit of the recent morphofunctional techniques such as transmitters visualization. The study of chemically-defined neurons led to a better understanding of the inner retinal circuitry, and especially of amacrine cells. Most of the well-known neurotransmitters have been localized in different subclasses of amacrine cells which are involved in the modulation of $\mathrm{ON}$ and/or OFF circuits, depending on the sublayer of the inner plexiform layer where they establish connections. In pathological states, the final output from the retina to the visual centers can be modified according to the amacrine subclass whose transmitter metabolism is disturbed. However, dopamine seems to have multiple functions in the retina, and they are mainly under the control of GABA and depending upon the light/dark cycle. Dopamine is to be considered as a retinal modulator more than a transmitter. 